\title{
Development of 1-2 years Offspring Born to Mothers with Polycystic Ovary Syndrome
}

\author{
Yuanlin Wang ${ }^{1}$, Lanzhong Guo ${ }^{2}$, Jun Jiang ${ }^{2}$, Fangfang Wang ${ }^{1}$, Paul J. Hardiman ${ }^{3}$ and Fan $\mathrm{Qu}^{1}$ \\ ${ }^{1}$ Women's Hospital, School of Medicine, Zhejiang University, Hangzhou, China \\ ${ }^{2}$ Dongyang Women's and Children's Hospital, Dongyang, China \\ ${ }^{3}$ Institute for Women's Health, University College London, London, United Kingdom
}

\begin{abstract}
Objective: To evaluate the body mass index and neurologic development of 1-2 years offspring born to mothers with polycystic ovary syndrome.

Study Design: A case-control study.

Place and Duration of Study: Dongyang Women and Children's Hospital, Zhejiang Province, China, between June 2018 and January 2019.

Methodology: A total of 145 children were included in the final analysis, including 16 daughters of mothers with PCOS, 13 sons of mothers with PCOS, 55 daughters of mothers without PCOS and 61 sons of mothers without PCOS. Developmental assessments for each child were conducted, including anthropometric measurements and ability developments using the Denver developmental screening test.

Results: The body mass index of children was significantly lower in polycystic ovary syndrome group than control group $(p=0.022)$. Children of mothers with polycystic ovary syndrome and control group had no significant differences in the outcomes of the Denver developmental screening test (all $p>0.05$ ).

Conclusion: Maternal polycystic ovary syndrome may affect body mass index of offspring aged 1-2 years and had no negative effects on neurologic development. However, this conclusion may be limited due to the small sample size.
\end{abstract}

Key Words: Neurologic development, Polycystic ovary syndrome, Offspring, Denver development screening test, Body mass index.

How to cite this article: Wang Y, Guo L, Jiang J, Wang F, Hardiman PJ, Qu F. Development of 1-2 years Offspring Born to Mothers with Polycystic Ovary Syndrome. J Coll Physicians Surg Pak 2021; 31(10):1186-1190.

\section{INTRODUCTION}

Polycystic ovary syndrome (PCOS) is the most common endocrine disorder in women of reproductive age, with an incidence of $15 \%$ based on the Rotterdam criteria. ${ }^{1}$ According to the 'developmental origins of health and disease' concept, a foetus in an adverse intrauterine environment will result in a series of integrated responses to adapt to the future environment, which are associated with health problems in later adult life. ${ }^{2}$ There is increasing evidence indicating that women with PCOS may have a high level of androgen concentrations in the intrauterine environment during pregnancy, ${ }^{3}$ which may lead to their offspring's adverse health outcomes. ${ }^{4}$ Human studies have proposed that offspring of PCOS women show abnormalities in growth and development, and in cardiovascular, metabolic and reproductive systems. ${ }^{5-7}$

Correspondence to: Dr. Fan Qu, Women's Hospital, School of Medicine, Zhejiang University, Xueshi Road, Hangzhou, Zhejiang, 310006, China

E-mail: syqufan@zju.edu.cn

Received: May 12, 2021; Revised: August 31, 2021; Accepted: September 22, 2021

DOI: https://doi.org/10.29271/jcpsp.2021.10.1186
However, there are different conclusions concerning the physical development of offspring born to PCOS women compared with control groups. Although most studies have focused on physical development, few studies have assessed the neurologic and language development of offspring born to PCOS women. However, prenatal androgen exposure may be involved in the development of the mammalian brain and cause long-term changes in sexually dimorphic behaviour and cognitive function, increasing the risk of offspring developing anxiety disorders. ${ }^{8,9}$

The Denver developmental screening test (DDST), an assessment tool for developmental screening, has been utilised worldwide to evaluate the developmental status of children in general (e.g. language, motor, cognition and sociality), to identify developmental delays in asymptomatic children early; and to diagnose developmental impairment. ${ }^{10}$ In line with these considerations, the authors guessed maternal PCOS may affect the neurologic development of offspring.

The aim of this study was to evaluate the body mass index, and the neurologic development of 1-2 years offspring born to mothers with PCOS using the DDST. 


\section{METHODOLOGY}

This case-control study was conducted between June 2018 and January 2019 in Dongyang Women and Children's Hospital, Zhejiang Province, China. The study was approved by the Ethics Committee of Women and Children's Hospital, Dongyang, China, and written informed consents were obtained from all the children's parents.

All the mothers' baseline characteristics and their children's clinical characteristics at birth were collected from the medical records of the hospital. The PCOS women before pregnancy were diagnosed according to the Rotterdam diagnostic criteria for PCOS (2003). ${ }^{11}$ As control mothers, the authors selected women of similar socio-economic level as the PCOS women, with a history of regular 28- to 32-day menstrual cycles, without hirsutism and other manifestations of hyperandrogenism. Children were excluded from analysis, if they were not born in a singleton pregnancy, less than 37-week gestational age, less than 2500-g birth weight or with missing data, and those with a history of birth asphyxia and congenital diseases. A total of 145 children were included in the final analysis, including 16 daughters of mothers with PCOS, 13 sons of mothers with PCOS, 55 daughters of mothers without PCOS, and 61 sons of mothers without PCOS. Each child born to PCOS women was assigned to four children born to control women based on matching for age.

For developmental assessment of 1-2 years children, the authors evaluated physical development by anthropometric measurements, and neurologic development by DDST. Two trained professionals independently completed all the assessments for all the children.

Anthropometric measurements: These included body mass index (BMI) and head circumference. BMI was calculated by weight $(\mathrm{Kg}) /$ height $^{2}(\mathrm{~m})$.

The DDST is used for the early identification of developmental delays in children from zero to six years of age. The standardised Chinese edition was used in the present study. ${ }^{12}$ The standardised DDST consists of 104 items, divided into four parts: (a) personal-social; (b) fine motor-adaptive; (c) language; and (d) gross motor. Each item can be scored by the response of 'passes', 'fails', 'refuses' or 'has not had the opportunity'. The authors used normal (no delays), suspect ( 2 or more caution items and/or 1 or more delays), abnormal ( 2 or more delays) or untestable (refusals of one or more items completely to the left of the age line or more than one item intersected by the age line in the $75-90 \%$ area) as the label of the results. ${ }^{13}$

Statistical analysis was performed using IBM SPSS Statistics version 23.0 (IBM Corp., Armonk, NY, USA); where continuous variables were normally distributed, the independent Student'st-test was used, and the data expressed as means \pm standard deviations. For the comparison of categorical variables, the Chi-square test was used, and the data were presented as numbers and percentages. Binary logistic regression analysis was also performed to assess the potential associations between maternal PCOS and outcomes of DDST in offspring. A value of $p<0.05$ was considered statistically significant.
The confounding bias was controlled by age-matched design and regression analysis; and the measurement bias was controlled by evaluating the participants under double-blind conditions.

\section{RESULTS}

As shown in Table I, there were no significant differences between mothers with PCOS and control mothers with respect to maternal height, preconception BMI, employment status, educational level, parity, maternal weight gain, pregnancy complications, age at delivery, weight at term of delivery, BMI at term of delivery and mode of delivery (all $p>0.05$ ). No significant differences existed for gestational age, birth weight, Apgar score (1 minute), Apgar score ( 5 minutes) and age at evaluation between children of the PCOSgroup and control children (all p > 0.05, Table I).

Table I: Baseline characteristics of mothers and children.

\begin{tabular}{|c|c|c|c|}
\hline w & $P \cos (n=29)$ & Control $(n=116)$ & p-value \\
\hline \multicolumn{4}{|l|}{ Mothers } \\
\hline Height(cm) & $158.76 \pm 4.30$ & $159.36 \pm 4.47$ & 0.513 \\
\hline Preconception BMI $\left(\mathrm{kg} / \mathrm{m}^{2}\right)$ & $22.32 \pm 3.84$ & $21.23 \pm 2.78$ & 0.082 \\
\hline \multicolumn{4}{|l|}{ Current employment status [n(\%)] } \\
\hline Full-time employment & $6(20.70)$ & $28(24.10)$ & \multirow{3}{*}{0.920} \\
\hline Part-time employment & $17(58.60)$ & $64(55.20)$ & \\
\hline Registered unemployed & $6(20.70)$ & $24(20.70)$ & \\
\hline \multicolumn{4}{|l|}{ Educational level [n(\%)] } \\
\hline Primary/vocational & 0 & $2(1.70)$ & \multirow{3}{*}{0.584} \\
\hline Secondary & $13(44.80)$ & $60(51.70)$ & \\
\hline University & $16(55.20)$ & $54(46.60)$ & \\
\hline Parity & $1.38 \pm 0.49$ & $1.44 \pm 0.55$ & 0.590 \\
\hline Maternal weight gain $(\mathrm{kg})$ & $12.64 \pm 4.45$ & $13.70 \pm 3.44$ & 0.165 \\
\hline \multicolumn{4}{|l|}{ Pregnancy complication [n(\%)] } \\
\hline Yes & $4(13.80)$ & $9(7.80)$ & \multirow{2}{*}{0.513} \\
\hline No & $25(86.20)$ & $107(92.20)$ & \\
\hline Age at delivery (years) & $29.21 \pm 4.86$ & $28.41 \pm 5.00$ & 0.444 \\
\hline Weight at term of delivery $(\mathrm{kg})$ & $68.84 \pm 8.62$ & $67.64 \pm 8.41$ & 0.495 \\
\hline $\mathrm{BMI}$ at term of delivery $\left(\mathrm{kg} / \mathrm{m}^{2}\right)$ & $27.34 \pm 3.41$ & $26.62 \pm 3.01$ & 0.268 \\
\hline \multicolumn{4}{|l|}{ Mode of delivery [n(\%)] } \\
\hline Vaginal delivery & $16(55.20)$ & $67(57.80)$ & \multirow{2}{*}{0.801} \\
\hline Caesarean delivery & $13(44.80)$ & $49(42.20)$ & \\
\hline \multicolumn{4}{|l|}{ Chidren } \\
\hline Gestational age (weeks) & $39.15 \pm 1.30$ & $39.50 \pm 1.06$ & 0.133 \\
\hline Birth weight (g) & $3353.79 \pm 554.57$ & $3341.72 \pm 372.36$ & 0.912 \\
\hline \multicolumn{4}{|l|}{ Apgar score (1 minute) $[\mathrm{n}(\%)]$} \\
\hline$\geq 7$ & $29(100.00)$ & $116(100.00)$ & \multirow[b]{2}{*}{-} \\
\hline$<7$ & 0 & 0 & \\
\hline \multicolumn{4}{|l|}{ Apgar score (5 minute) [n(\%)]- } \\
\hline$\geq 7$ & $29(100.00)$ & $116(100.00)$ & \multirow[b]{2}{*}{ 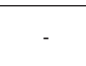 } \\
\hline$<7$ & 0 & 0 & \\
\hline Age at evaluation(months) & $21.83 \pm 2.71$ & $21.45 \pm 3.09$ & 0.546 \\
\hline
\end{tabular}

Table II: Developmentalassessment of children.

\begin{tabular}{|c|c|c|c|}
\hline Items & $P \operatorname{COS}(n=29)$ & Control $(n=116)$ & $p$-value \\
\hline BMI $\left(\mathrm{kg} / \mathrm{m}^{2}\right)$ & $15.84 \pm 1.23$ & $16.49 \pm 1.37$ & $0.022^{\circ}$ \\
\hline Head circumference $(\mathrm{cm})$ & $47.96 \pm 1.48$ & $48.03 \pm 1.53$ & 0.807 \\
\hline \multicolumn{4}{|l|}{ DDST } \\
\hline \multicolumn{4}{|l|}{ Personal-social [n(\%)] } \\
\hline Normal & $28(96.60)$ & $113(97.40)$ & \multirow{3}{*}{0.746} \\
\hline Suspicious & $1(3.40)$ & $2(1.70)$ & \\
\hline Abnormal & 0 & $1(0.70)$ & \\
\hline \multicolumn{4}{|l|}{ Fine motor $[n(\%)]$} \\
\hline Normal & $29(100.00)$ & $113(97.40)$ & \multirow{3}{*}{1.000} \\
\hline Suspicious & 0 & $3(2.60)$ & \\
\hline Abnormal & 0 & 0 & \\
\hline \multicolumn{4}{|l|}{ Language $[n(\%)]$} \\
\hline Normal & $29(100.00)$ & 104(89.70) & \multirow{3}{*}{0.195} \\
\hline Suspicious & 0 & $11(9.50)$ & \\
\hline Abnormal & 0 & $1(0.90)$ & \\
\hline \multicolumn{4}{|l|}{ Gross motor [n (\%)] } \\
\hline Normal & $29(100.00)$ & $110(94.80)$ & \multirow{3}{*}{0.457} \\
\hline Suspicious & 0 & $3(2.60)$ & \\
\hline Abnormal & 0 & $3(2.60)$ & \\
\hline
\end{tabular}


Table III: Associations among maternal PCOS, other potential confounders; and DDST outcomes in offspring

\begin{tabular}{|c|c|c|c|c|c|c|c|c|}
\hline & \multicolumn{2}{|l|}{ Personal-social } & \multicolumn{2}{|l|}{ Fine motor } & \multicolumn{2}{|l|}{ Language } & \multicolumn{2}{|l|}{ Gross motor } \\
\hline & OR $(95 \% \mathrm{Cl})$ & p-value & OR $(95 \% \mathrm{Cl})$ & p-value & OR $(95 \% \mathrm{Cl})$ & p-value & OR $(95 \% \mathrm{Cl})$ & p-value \\
\hline Maternal PCOS & $1.77(0.02-140.13)$ & 0.80 & - & 0.99 & - & 0.99 & - & 0.99 \\
\hline Preconception BMI & $1.24(0.80-1.93)$ & 0.32 & $1.17(0.55-2.48)$ & 0.67 & $1.07(0.84-1.36)$ & 0.56 & $1.17(0.83-1.64)$ & 0.35 \\
\hline Maternal employment status & - & 0.73 & - & 1.00 & - & 0.47 & - & 0.31 \\
\hline Maternal educational level & - & 0.30 & - & 1.00 & - & 0.75 & - & 0.75 \\
\hline Pregnancy complication & - & 0.99 & $0.20(0.00-62.56)$ & 0.58 & $1.09(0.09-12.57)$ & 0.94 & - & 0.99 \\
\hline Maternal age at delivery & $0.98(0.72-1.35)$ & 0.94 & $1.03(0.60-1.78)$ & 0.89 & $0.97(0.84-1.12)$ & 0.73 & $1.13(0.94-1.34)$ & 0.17 \\
\hline Mode of delivery & - & 0.99 & $3.91(0.00-3740.82)$ & 0.69 & $1.92(0.38-9.57)$ & 0.42 & $1.08(0.14-7.96)$ & 0.93 \\
\hline Children gender & $0.43(0.00-29.80)$ & 0.69 & $2.79(0.02-267.15)$ & 0.65 & $3.16(0.77-12.96)$ & 0.10 & $0.60(0.08-4.52)$ & 0.62 \\
\hline Birth weight & $0.99(0.99-1.00)$ & 0.14 & $0.99(0.98-1.00)$ & 0.09 & $0.99(0.99-1.00)$ & 0.53 & $1.00(0.99-1.00)$ & 0.36 \\
\hline
\end{tabular}

As shown in Table II, the BMI of children was significantly lower in the PCOS group than the control group ( $p=0.022)$, and no significant differences existed for head circumference, DDST outcomes (including personal-social, fine motoradaptive, language and gross motor) between children of the PCOS group and control children (all $p>0.05$ ).

As shown in Table III, there were no significant associations between maternal PCOS and DDST outcomes ( $p>0.05)$, after adjusting the potential confounders including preconception BMI, maternal employment status, maternal educational level, pregnancy complication, maternal age at delivery, mode of delivery, children gender and birth weight.

\section{DISCUSSION}

In the present study, the offspring of PCOS mothers had lower BMI at the age of 1-2 years. Current data did mention that maternal PCOS may affect physical development of offspring. A single-centre prospective cohort study in Denmark suggested that maternal PCOS may increase the risk of obesity in three-year offspring.$^{14}$ Crisosto et al. found the total cholesterol and low-density lipoprotein levels of male offspring of PCOS mothers increased during the puberty period. ${ }^{15}$ These results were inconsistent with two studies, but it should be noted that the offspring in this study was much younger than the others in other studies. The trend of obesity in children born to PCOS mothers may be obvious as they getting older. However, the use of medication such as metformin had an impact on the weight of offspring born to PCOS mothers. ${ }^{16}$ Information concerning the use of metformin for PCOS was not available in this study, which may have biased the results. Apart from treatment with medication, family parenting environment may be one reason that offspring of PCOS women showed lower BMI.

Personal-social, fine motor, language and gross motor abilities of 1-2 years offspring born to mothers with PCOS were assessed by DDST and in the offspring of control mothers. Maternal pre-pregnancy obesity was reported to increase the risk of psychiatric problems and neurocognitive developments of offspring. ${ }^{17}$ In addition, children's brains, cognitive and emotional development are closely related to their surrounding environments after birth. A study showed the infants' caregivers had a dominant role in the physical and cognitive development of infants in China. ${ }^{13}$ Maternal educa- tion had an impact on children' growth and development, and higher levels of maternal education were positively associated with children's cognitive development. ${ }^{18}$ An Icelandic study suggested that an association exists between a number of factors such as low birth weight, young age of the mother at the time of the child's birth and caesarean section and attention deficit and hyperactivity disorder (ADHD) in offspring. ${ }^{19}$ Other confounding variables, including children gender and pregnancy complication have also been proposed as risk factors of autism spectrum disorder (ASD) in offspring. ${ }^{20,21}$ Considering these possible confounders, logistic regression analysis was performed to minimise the effects of confounding biases. However no association existed between maternal PCOS and outcomes of DDST. The results of the present authors were inconsistent with a previous study which proposed PCOS progeny as being at increased risk for developmental delay when the age is less than 36 months, ${ }^{22}$ and other studies that found offspring of PCOS mothers seem to have a higher risk of developing neurodevelopmental disorders. ${ }^{23,24}$ Neuropsychiatric disorders in offspring born to mothers with PCOS was related to a high level of androgens in their utero, ${ }^{9,25}$ The present study did not collect the data concerning maternal serum androgen levels during pregnancy. In addition, the missed information on treatment for hyperandrogenaemia may have used before or during pregnancy may have biased the results in this study. Mothers who received treatment for PCOS were reported to have lower risks of having children with developmental delay compared to mothers who received no treatment. ${ }^{22}$

The case-control design was able to match a variety of confounding parameters between groups of children. However, the limit on the size of the group with PCOS and lacking of data regarding method of conception may lead to some bias and deviation. Further studies with a larger sample are needed to examine the association between PCOS and developmental outcomes of offspring born to PCOS mothers.

\section{CONCLUSION}

Maternal PCOS may affect BMI of offspring aged 1-2 years, and had no negative effects on neurologic development. However, the conclusion may be limited due to the small sample size. 


\section{FUNDING:}

This study was supported by the National Natural Science Foundation of China (81874480; 81873837), the Zhejiang Province Science Foundation for Distinguished Young Scholars (LR16H0 40001).

\section{ETHICAL APPROVAL:}

The study was approved by the Ethics Committee of Women and Children's Hospital, Dongyang, China.

\section{PATIENTS' CONSENT:}

Written informed consents were obtained from all the children's parents.

\section{CONFLICT OF INTEREST:}

The authors declared no conflict of interest.

\section{AUTHORS' CONTRIBUTION:}

YW: Study conception and design, analysis and interpretation of data, drafting the article and revising it critically for important intellectual content.

LG: Study conception and design, analysis and interpretation of data, drafting the article and revising it critically for important intellectual content.

$\mathrm{JJ}:$ Analysis, interpretation of data and revising it critically for important intellectual content and final approval of the version to be published.

FW: Interpretation of data and revising it critically for important intellectual content and final approval of the version to be published.

$\mathrm{PJH}$ : Conception of the work; revising the article critically for important intellectual content and final

approval of the version to be published.

FQ: Study conception and design, analysis and interpretation of data, and revising it critically for important intellectual content.

All authors read and approved the final manuscript. and agreed to be accountable for all aspects of the work.

\section{REFERENCES}

1. Fauser BC, Tarlatzis BC, Rebar RW, Legro RS, Balen AH, Lobo $\mathrm{R}$, et al. Consensus on women's health aspects of polycystic ovary syndrome (PCOS): The amsterdam ESHRE/ASRM-Sponsored 3rd PCOS consensus workshop group. Fertil Steril 2012; 97(1):28-38 e25. doi: 10.1016/j. fertnstert.2011.09.024.

2. Gluckman PD, Hanson MA, Beedle AS. Early life events and their consequences for later disease: A life history and evolutionary perspective. Am J Human Biol 2007; 19(1): 1-19. doi: 10.1002/ajhb.20590.

3. Filippou $\mathrm{P}$, Homburg R. Is foetal hyperexposure to androgens a cause of PCOS? Hum Reprod Update 2017; 23(4):421-32. doi: 10.1093/humupd/dmx013.

4. Puttabyatappa M, Cardoso RC, Padmanabhan V. Effect of maternal PCOS and PCOS-like phenotype on the offspring's health. Mol Cell Endocrinol 2016; 435:29-39. doi: 10.1016/j. mce.2015.11.030.
5. Wilde MA, Eising JB, Gunning MN, Koster MPH, Evelein AMV, Dalmeijer GW, et al. Cardiovascular and metabolic health of 74 children from women previously diagnosed with polycystic ovary syndrome in comparison with a population-based reference cohort. Rep Sci 2018; 25(10):1492-500. doi: 10.1177/1933719117749761.

6. Kent SC, Gnatuk CL, Kunselman AR, Demers LM, Lee PA, Legro RS. Hyperandrogenism and hyperinsulinism in children of women with polycystic ovary syndrome: A controlled study. J Clin Endocrinol Metab 2008; 93(5): 1662-9. doi: 10.1210/jc.2007-1958.

7. Sir-Petermann T, Codner E, Perez V, Echiburu B, Maliqueo M, Ladron de Guevara A, et al. Metabolic and reproductive features before and during puberty in daughters of women with polycystic ovary syndrome. J Clin Endocrinol Metab 2009; 94(6):1923-30. doi: 10.1210/jc.2008-2836.

8. Manson JE. Prenatal exposure to sex steroid hormones and behavioral/cognitive outcomes. Metabolism 2008; 57 Suppl 2:S16-21. doi: 10.1016/j.metabol.2008.07.010.

9. Hu M, Richard JE, Maliqueo M, Kokosar M, Fornes R, Benrick $A$, et al. Maternal testosterone exposure increases anxiety-like behavior and impacts the limbic system in the offspring. Proc Natl Acad Sci USA 2015; 112(46):14348-53. doi: 10.1073/pnas.1507514112.

10. Jeong SU, Kim GC, Jeong HJ, Kim DK, Hong YR, Kim HD, et al. The validity of the bayley-III and DDST-II in preterm infants with neurodevelopmental impairment: A pilot study. Ann Rehab Med 2017; 41(5):851-7. doi: 10.5535/arm. 2017.41. 5.851 .

11. Rotterdam EA-SPcwg. Revised 2003 consensus on diagnostic criteria and long-term health risks related to polycystic ovary syndrome (PCOS). Hum Reprod 2004; 19(1):41-7. doi: 10.1093/humrep/deh098.

12. Lin CJ, Su ZY, Li JP. Denver's intelligent development screening renormalises among normal children in six cities of China. Chinese J Pedia 1983; 21(4):206-9.

13. Li Q, Liang F, Liang W, Zhang J, Niu M, Han Y. The Influence of Different Caregivers on Infant Growth and Development in China. Frontiers Pedia 2017; 5:243. doi: 10.3389/fped. 2017.00243.

14. Finnbogadottir SK, Glintborg D, Jensen TK, Kyhl HB, Nohr EA, Andersen $M$. Insulin resistance in pregnant women with and without polycystic ovary syndrome, and measures of body composition in offspring at birth and three years of age. Acta obstetricia et gynecologica Scandinavica 2017; 96(11):1307-14. doi: 10.1111/aogs.13200.

15. Crisosto N, Echiburu B, Maliqueo M, Luchsinger M, Rojas $P$, Recabarren $\mathrm{S}$, et al. Reproductive and metabolic features during puberty in sons of women with polycystic ovary syndrome. Endocr Connect 2017; 6(8):607-13. doi: 10.1530/EC-17-0218.

16. Hanem LGE, Stridsklev S, Juliusson PB, Salvesen O, Roelants $M$, Carlsen SM, et al. Metformin use in PCOS pregnancies increases the risk of offspring overweight at 4 years of age: Follow-up of two RCTs. J Clin Endocrinol Metab 2018; 103(4):1612-21. doi: 10.1210/jc.2017-02419.

17. Alvarez-Bueno C, Cavero-Redondo I, Lucas-de la Cruz L, Notario-Pacheco B, Martinez-Vizcaino V. Association between pre-pregnancy overweight and obesity and chil- 
dren's neurocognitive development: A systematic review and meta-analysis of observational studies. Int J Epidemiol 2017; 46(5):1653-66. doi: 10.1093/ije/dyx122.

18. Harding JF. Increases in maternal education and low-income children's cognitive and behavioral outcomes. Dev Psychol 2015; 51(5):583-99. doi: 10.1037/a0038920.

19. Valdimarsdottir $M$, Hrafnsdottir $A H$, Magnusson $P$, Gudmundsson 00. The frequency of some factors in pregnancy and delivery for Icelandic children with ADHD. Laeknabladid 2006; 92(9):609-14.

20. Knickmeyer RC, Baron-Cohen S. Fetal testosterone and sex differences in typical social development and in autism. J Child Neurol 2006; 21(10):825-45. doi: 10.1177/088307 38060210101601.

21. Gardener H, Spiegelman D, Buka SL. Prenatal risk factors for autism: Comprehensive meta-analysis. BrJ Psychiatry 2009; 195(1):7-14. doi: 10.1192/bjp.bp.108.051672.

22. Bell GA, Sundaram R, Mumford SL, Park H, Mills J, Bell EM, et al. Maternal polycystic ovarian syndrome and early offspring development. Hum Reprod 2018; 33(7):1307-15. doi: 10.1093/humrep/dey087.

23. Kosidou K, Dalman C, Widman L, Arver S, Lee BK, Magnusson $C$, et al. Maternal polycystic ovary syndrome and the risk of autism spectrum disorders in the offspring: a population-based nationwide study in Sweden. Mol Psychiatry 2016; 21(10):1441-8. doi: 10.1038/mp.2015. 183.

24. Kosidou K, Dalman C, Widman L, Arver S, Lee BK, Magnusson $C$, et al. Maternal polycystic ovary syndrome and risk for Attention-Deficit/Hyperactivity disorder in the offspring. Biol Psychiatry 2017; 82(9):651-9. doi: 10.1016/j. biopsych.2016.09.022.

25. Cesta CE, Oberg AS, Ibrahimson A, Yusuf I, Larsson H, Almqvist $C$, et al. Maternal polycystic ovary syndrome and risk of neuropsychiatric disorders in offspring: Prenatal androgen exposure or genetic confounding? Psychol Med 2019:1-9. 